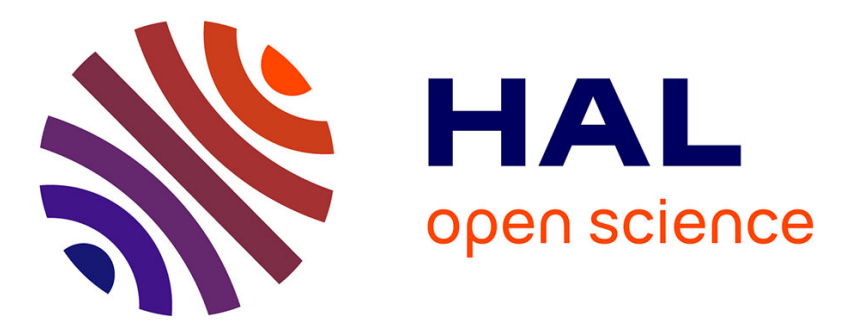

\title{
Influence of feedback on modal shapes and its consequences on broadband energy harvesting
}

\author{
C Giraud-Audine, Frédéric Giraud, M Amberg
}

\section{To cite this version:}

C Giraud-Audine, Frédéric Giraud, M Amberg. Influence of feedback on modal shapes and its consequences on broadband energy harvesting. Joint Ieee International Symposium on ISAF IWATMD PFM, May 2014, PennState University, United States. pp.4, 10.1109/ISAF.2014.6922971 . hal01254893

\section{HAL Id: hal-01254893 \\ https://hal.science/hal-01254893}

Submitted on 12 Jan 2016

HAL is a multi-disciplinary open access archive for the deposit and dissemination of scientific research documents, whether they are published or not. The documents may come from teaching and research institutions in France or abroad, or from public or private research centers.
L'archive ouverte pluridisciplinaire HAL, est destinée au dépôt et à la diffusion de documents scientifiques de niveau recherche, publiés ou non, émanant des établissements d'enseignement et de recherche français ou étrangers, des laboratoires publics ou privés. 


\section{Influence of feedback on modal shapes and its consequences on broadband energy harvesting}

\author{
C. Giraud-Audine \\ Laboratoire d'Electrotechnique et d'Electronique de \\ Puissance \\ Arts et Métiers Paristech \\ Lille, France \\ christophe.giraud-audine@ensam.eu
}

\author{
F. Giraud and M. Amberg \\ Laboratoire d'Electrotechnique et d'Electronique de \\ Puissance \\ University Lille 1 \\ Villeneuve d'Ascq, France
}

\begin{abstract}
We study the effect of feedback on an energy harvester for broadband applications. Using modal analysis we show that it has an influence on modal shape as a side effect of the resonance shifting. We show that the piezoelectric coupling can thus be reduced. Experimental results are in agreement and also reveal that non-linear effects are promoted in some cases.
\end{abstract}

Piezoelectric; broadband energy harvesting; modal analysis;

\section{INTRODUCTION}

Energy harvesting from ambient vibrations is considered as one solution to provide energy to autonomous system requiring low power or working intermittently [1]. Resonance is the optimal condition for energy harvesting because elastic stress is compensated by inertia forces. However, in practice the resonance condition is rarely verified. First, the mechanical source usually generates vibrations in a bandwidth rather than a single frequency during operation. Second, the parameters of the harvester can vary resulting in a shift of its own resonance frequency, hence the ongoing research on broadband harvesters [2].

Another possibility is to use the inverse piezoelectric effect to compensate the elastic stress or the inertia $[3,4]$. The resonance can then be shifted thus enabling to harvest energy on a larger bandwidth. The contribution of this paper is to show that proportional feedback using the measurement of the tip displacement can modify both the frequencies and the modal shape. However, we show that the coupling factor of the harvester will also be impacted.

The paper is structured as follow: in next section, the model is briefly presented. In the third section, modal bimorph bender with feedback is analyzed. Finally the model is compared to experimental tests.

\section{MODEL}

\section{A. Assumption and notations}

The schematic of Fig 1 depicts a simplified model of a slender bender used for energy harvesting. The problem is supposed to be bidimensionnal that is the different fields are

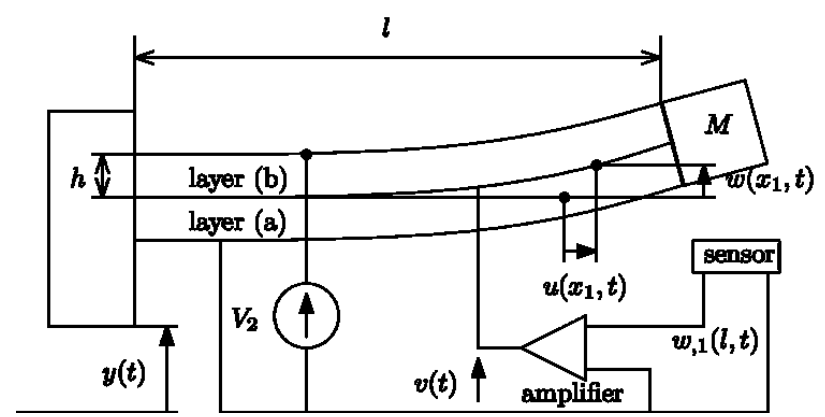

Figure 1. schematic of the studied system

not dependent on $x_{2}$ (direction orthogonal to the plane of the schematic). The bender is constituted by two piezoelectric (a and $b$ ) layers polarized to operate in 31 mode. The end at $x_{l}=0$ is clamped to the moving frame while a proof mass $M$ is clamped at the free end $\left(x_{I}=l\right)$. The moving frame is translated along the direction $\mathbf{e}_{3}$, its position relative to the reference frame is $y(t)$. The bender is supposed to be mechanically unloaded, apart from the effect of inertia.

The electrodes are connected as shown. The voltage $V_{2}$ is constant while the voltage $v$ is varied accordingly to some measurement of the end tip. We consider small displacements and since electric potentials are imposed on the electrodes, it will also be assumed that the electrical field is mainly along the $\mathbf{e}_{3}$ direction.

\section{B. Piezoelectric}

For the following discussion, it is convenient to consider displacement and voltage to formulate the problem thus the relevant piezoelectric equation used throughout this paper are :

$$
\begin{aligned}
& \mathbf{T}=\mathbf{c}^{\mathbf{E}} \mathbf{S}-\mathbf{e}^{\mathrm{T}} \mathbf{E} \\
& \mathbf{D}=\mathbf{e} \mathbf{S}+\boldsymbol{\varepsilon}^{\mathbf{S}} \mathbf{E}
\end{aligned}
$$

$\mathbf{c}^{\mathbf{E}}$ is the rigidity matrix at constant electric field, $\mathbf{e}$ is the piezoelectric matrix, and $\boldsymbol{\varepsilon}^{\mathbf{S}}$ is the permittivity at constant strain. The corresponding thermodynamic potential is [5] :

$$
\mathrm{dG}_{2}(\mathbf{S}, \mathbf{E})=\mathbf{T}^{\mathrm{T}} \mathrm{d} \mathbf{S}-\mathbf{D}^{\mathrm{T}} \mathrm{d} \mathbf{E}
$$

This work has been carried out within the framework of the Mint project of Inria (France) and the StimTac Project of the Institut de Recherche sur les Composantans logIciels et matériels pour la Communication Avancée (IRCICA) 


\section{Kinematic}

The bonding of the layers is supposed to be perfect, thus a continuous displacement field is considered. In this study shear strain is not considered, the bender being thin. The deformation considered consists in the superposition of an extension strain and a flexion. Thus, following the classical Bernouilli beam theory $[5,6]$, the displacement is given by:

$$
\mathbf{u}\left(x_{1}, x_{3}, t\right)=\left\{u\left(x_{1}, t\right)-x_{3} w_{1}\left(x_{1}, t\right), w\left(x_{1}, t\right)+y(t)\right\}^{\mathrm{T}}
$$

Which results in a strain field:

$$
\mathbf{S}\left(x_{1}, x_{3}, t\right)=\left\{u_{, 1}\left(x_{1}, t\right)-x_{3} w_{, 11}\left(x_{1}, t\right), 0,0,0,0,0,0\right\}^{\mathrm{T}}
$$

\section{Electrostatic}

Considering now the electrical displacement D, the condition $\operatorname{div} \mathbf{D}=0$ must hold. According to the piezoelectric law (1) and the strain (4), and neglecting the component of the electric field along $\mathbf{e}_{1}$, the electrical potential in one the piezoelectric of the bender is given by:

$$
\phi_{i}\left(x_{1}, x_{3}, t\right)=-\frac{e_{31}}{\varepsilon_{33}^{s}} w_{, 11}\left(x_{1}, t\right) \frac{x_{3}^{2}}{2}+A_{i}\left(x_{1}, t\right) x_{3}+B_{i}\left(x_{1}, t\right)
$$

The functions $A_{i}\left(x_{1}, t\right), B_{i}\left(x_{1}, t\right)$, where $i \in\{a, b\}$ denote the two piezoelectric domains, are functions to be determined from the potential on the electrodes.

\section{E. Dynamic Equations}

The augmented Lagrangian of the piezoelectric energy harvester is given by:

$$
\begin{aligned}
& L=\frac{1}{2} \iiint \rho \dot{\mathbf{u}}^{\mathrm{T}} \dot{\mathbf{u}}-G_{2} d \Omega+\frac{1}{2} M \dot{\mathbf{u}}_{x_{1}=l}^{\mathrm{T}} \dot{\mathbf{u}}_{x_{1}=l}+F y+ \\
& +\left(\frac{V_{2}}{2}+v\right)\left(Q_{1}-Q_{2}\right)+V_{2} Q_{2}
\end{aligned}
$$

where the four last contributions corresponds to the mechanical energy due to the external force, the kinetic energy of the proof mass and the energy supplied by the generators. Applying variation calculus, the following equations for the extension and the flexion displacement field respectively are obtained:

$$
\begin{aligned}
& -\mu \ddot{m}\left(x_{1}, t\right)+K u_{, 11}\left(x_{1}, t\right)=0 \\
& -\mu \ddot{m}\left(x_{1}, t\right)+J \ddot{w}_{, 11}\left(x_{1}, t\right)-D w_{, 111}\left(x_{1}, t\right)-\mu \ddot{y}(t)=0
\end{aligned}
$$

where, $\mu$ is the mass per unit length, $J$ is the rotational inertia per unit length, $K$ is the compressive rigidity, $D$ is equivalent the flexural rigidity. $w_{, i}$ denotes the derivative with respect to $x_{i}$, dots are time derivatives. The mechanical boundary conditions at the free end are:

$$
\left\{\begin{array}{c}
K u_{, 1}(l, t)=-\kappa_{e x} V_{2}(t) \\
D w_{, 11}(l, t)=\kappa_{f l}(l, t) \\
D w_{, 111}(l, t)-J \dot{w}_{, 1}(l, t)=M(\ddot{w}(l, t)+\ddot{y}(t))
\end{array}\right.
$$

Where $\kappa_{e x}=e_{31} a$ and $\kappa_{f l}=e_{31} a h$ are the piezoelectric coefficients for extension and flexion respectively,. At the clamped end of the bender, the displacements are imposed:

$$
u(0, t)=w(0, t)=w_{, 1}(0, t)=0
$$

\section{THEORETICAL STUDY}

\section{A. Secular Equation}

In practice $V_{2}$ is constant to apply a pre-stress during bending. Therefore, $u\left(x_{1}, t\right)$ is constant and will not be considered in the following. Besides, the rotational inertia effects in (7) will not be considered in this study. To isolate the effect of feedback, $M$ is not considered. The equilibrium equation (7) with boundary conditions (8), and (9) are used to form the set of conditions that will lead to the modal analysis of the bender. Being an external excitation, $y(t)$ is set to zero [6]. Measurement of the displacement is realized by a sensing ceramic followed by an integrator. The resulting voltage is proportional to the deformation at the end of the bender. To realize a feedback, the applied voltage is proportional to the measurement voltage:

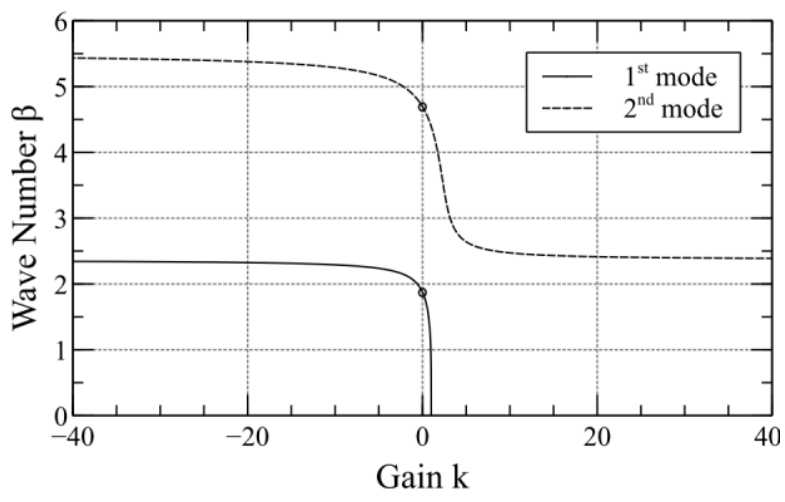

Figure 2. Wave number $\beta$ versus global feedback gain $\mathrm{k}$

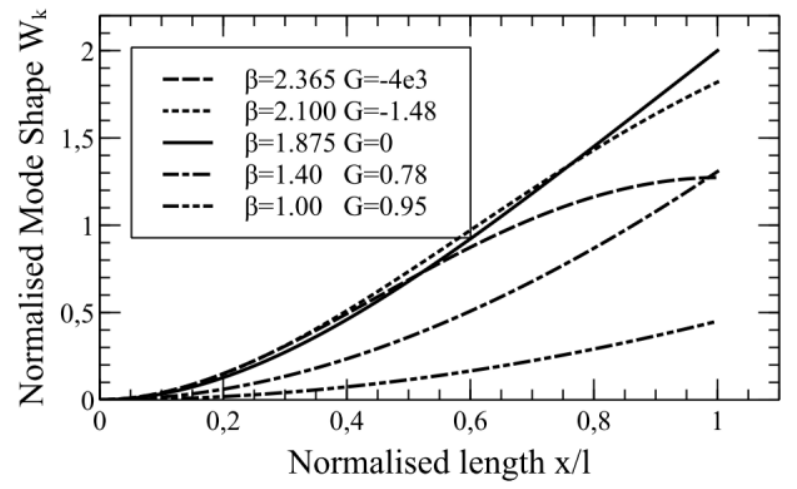

Figure 3. Mode shape for different feedback gain $\mathrm{k}$

$$
v(t)=G w_{, 1}(L, t)
$$

where $\mathrm{G}$ is the feedback gain. Using the set of boundary conditions (8) and (9) leads to the secular equation:

$$
\beta(1+\cos \beta \cosh \beta)-k(\cosh \beta \sin \beta+\cos \beta \sinh \beta)=0
$$


with $k=\kappa_{f l} G l$ the effective feedback gain. This differs from the classical secular equation of the clamped-free beam by its second terms induced by the feedback, which can be retrieved by letting $k=0$. The wave number and the angular frequency are related by the dispersion relation:

$$
\mu L^{4} \omega^{2}-D \beta^{4}=0
$$

\section{B. Resonant Frequencies and Modal Shapes}

Eq. (11) means that the values of $\beta$ can be modified by the gain $k$ as shown on Fig. 2 . The curves present asymptotes for $\beta$ such that $\tan \beta+\tanh \beta=0$ between the modes. A softening or a hardening effect depending on the sign of $\mathrm{k}$ can be obtained. Indeed, considering the first mode which is of special interest for energy harvesting, the wave number will tend toward 2.635 for an infinitely negative, so the ratio between the clamped free resonance frequency and the closed loop resonance frequency is limited to 1.588. A positive gain is more effective. Indeed, a variation form 0 to 1 would theoretically decrease $\beta$ from 1.875 to 0 . For $k=1$ a bifurcation occurs. The eigenvectors can be determined from (8) to (10), and the solutions $\beta_{k}$ to (11) up to a multiplicative constant. In the case at hand they write:

$$
\begin{array}{r}
W_{k}\left(x_{1}\right)=A_{k}\left[\cosh \frac{\beta_{k} x}{l}-\cos \frac{\beta_{k} x}{l}+\right. \\
\left.+\frac{\sin \beta_{k}-\sinh \beta_{k}}{\cos \beta_{k}+\cosh \beta_{k}}\left(\sinh \frac{\beta_{k} x}{l}-\sin \frac{\beta_{k} x}{l}\right)\right]
\end{array}
$$

The modes are orthogonal and can be normalized. $A_{k}$ are determined to verify:

$$
\mu \int W_{k}^{2}\left(x_{1}\right) d x_{1}=1
$$

Fig. 3 represents mode shapes according to the wave number and the corresponding feedback gain. For negative gain, the shapes tend toward a clamped-blocked rotation shape.

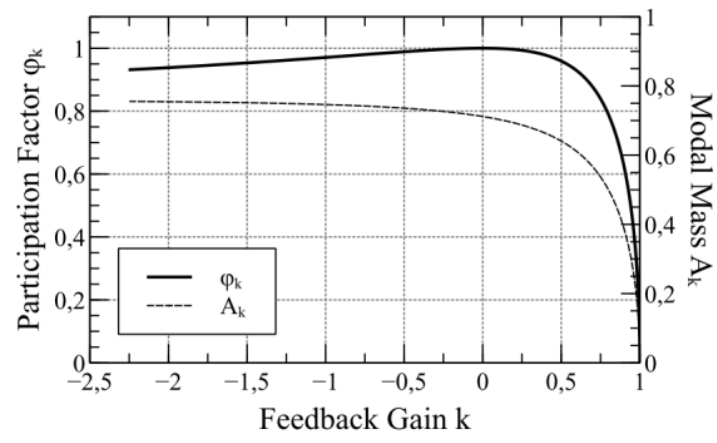

Figure 4. Participation factor and modal mass vs gain $\mathrm{k}$

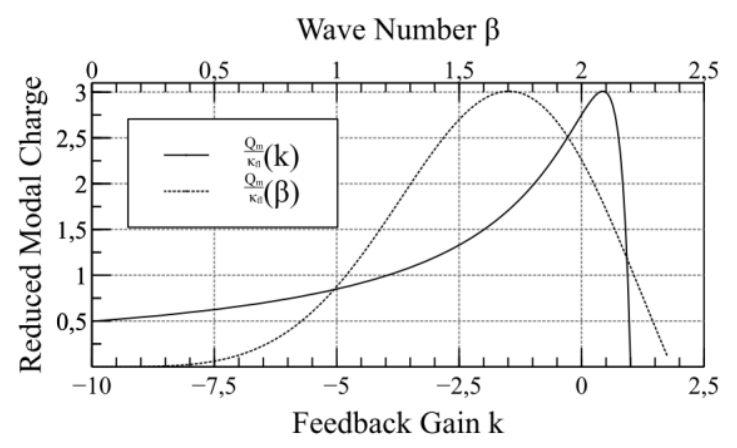

Figure 5. Reduced induced charge vs gain $\mathrm{k} /$ wave number $\beta$

For increasing positive gain, the curvature becomes more pronounced, but the mode amplitude decreases. This is confirmed by Fig. 4 where the modal mass are represented vs the feedback gain.

\section{Modal Force}

As mentioned, the normalized modes form an orthonormal base. Eq (7) can be solved by projection on each mode. The forces are then transformed to modal forces. The mechanical load $\mu \ddot{y}$ in (7) is uniform, thus, the corresponding modal force is proportional to:

$$
\varphi_{k}=\int W_{k}\left(x_{1}\right) d x_{1}
$$

The amplitude are likely to be larger for high participation factor. Fig. 4 shows that for softening gains, the participation factor collapses, while for hardening gain, it remains relatively constant. This is similar to the evolution of the modal mass, presented on the same graph.

\section{Modal Electromechanical Coupling}

Using the piezoelectric equations (1), the strain field (4) and the electrical potential (5), the charges on the electrodes are obtained by integrating $\mathbf{D}$ on their surface. One obtains for $Q_{l}$ :

$$
Q_{1}(t)=\varepsilon_{33}^{s} \frac{a l}{h} V_{2}-\kappa_{f l}\left[w_{, 1}\left(x_{1}, t\right)\right]_{0}^{l}
$$

(16) can be decomposed into a charge involving the blocked capacitance, and a charge induced by the strain in the material $Q_{m}$ which is potentially harvested. On Fig. 5 the graphs represent the reduced induced charge $Q_{m} / \kappa_{f l}=\left\lfloor W_{k, 1}\right\rfloor_{l}$ versus the feedback gain and the wave number. This quantity is proportional to the modal electromechanical coupling [7]. On the softening side, the coupling first slightly increasing (due to the increased curvature) then rapidly collapses (as the modal mass decreases). On the hardening side, the effect is similar, because the moment blocks the free end rotation. This in turn degrades the electromechanical coupling as it depends on the difference of slopes at both ends. 


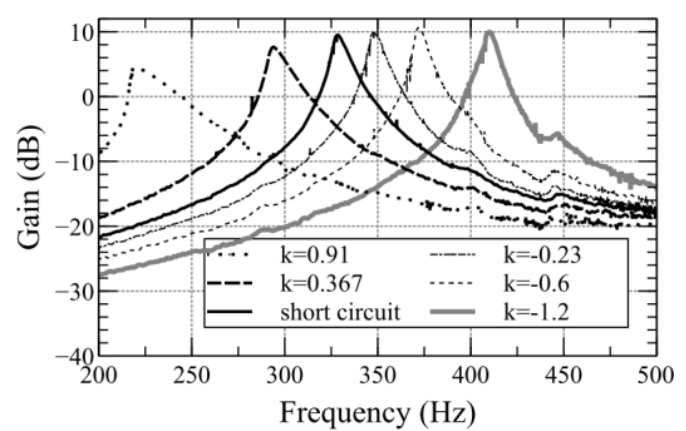

Figure 6. resonance for various experimental feedback gain

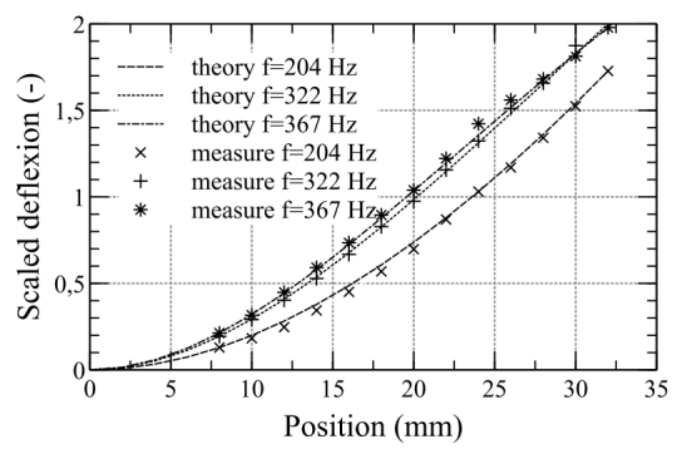

Figure 7. Experimental modal shapes

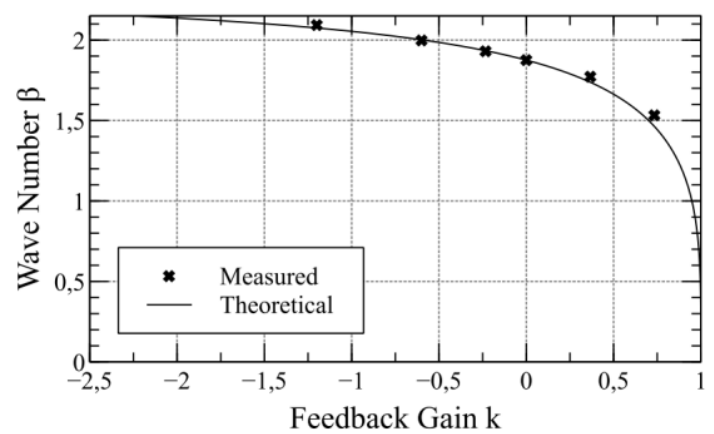

Figure 8. Theoretical and experimental wave number vs gain $\mathrm{k}$

\section{EXPERIMENTAL ASSESSMENT}

\section{A. Experimental setup}

The bender used for the experiments is a Noliac CMBP05 which consists in two bonded cofired monolithic ceramic $V_{2}$ is equal to $200 \mathrm{~V}$ and $v(t)$ varies from 0 to $200 \mathrm{~V}$ via the feedback loop. The deflexion is sensed using a Hall effect sensor. The measurement is integrated, then amplified by an analog amplifier (NF HSA 4052) to provide the necessary voltage superposed to a bias voltage. Vibrations are generated by a vibrating pot. Speed of the mobile is measured by a Polytec vibrometer (OFV5000, sensor OFV 505).

\section{B. Results}

The result for the magnitude (in $\mathrm{dB}$ ) between the vibrating pot's acceleration and the speed of the bender's tip is presented on Fig. 6 for various gains. The variation range of the gain was imposed by the voltage limitation for the hardening side (negatives gains) and instability of the closed loop on the softening side (positive gains) due to the bifurcation of the wave number as $k$ approaches 1 . As a consequence, the closed loop gain's sign changes with the inversion on the free end slope inducing a positive feedback. The resonant frequency can be shifted from $225 \mathrm{~Hz}$ to $410 \mathrm{~Hz}$. The short circuit resonance is obtained by setting $G=0 \quad\left(f_{c c}=329 \mathrm{~Hz}\right)$, therefore the percentage of variation $\left(f_{c l}-f_{c c}\right) / f_{c c}$ is $-32 \%$ to $25 \%$. For high positive values of $k$, non linearities become prominent because the feedback compensates the linear elasticity of the material.

The shapes were measured for some frequencies and compared to the predicted ones using (14) on Fig. 7. Since the amplitude is irrelevant, the graphs are scaled. The agreement between measurement and prediction confirm that the modal analysis is correct. To further assert the model, the measured and theoretical wave numbers are compared on Fig. 8. Again, a good agreement can be observed.

\section{CONCLUSION}

An analysis of a bender using a feedback for broadband harvesting has been proposed. The shifting of the resonant frequency is indeed possible within a range that was identified. However, it was shown that the potential benefit is in fact limited even though losses have not been considered in the study. Regarding softening, the mode are less easily excited by a uniform load that would result from the acceleration, this suggests a different shape of the bender for instance a varying section. Regarding hardening, as the bending moment tends to block the rotation at the free end, the coupling is degraded. Tests have confirmed these conclusions, although the electrical coupling could not be directly investigated due to the difficulty to measure the motional current. This point will be addressed in future works.

\section{REFERENCES}

[1] N. E. Dutoit, B. L. Wardle, and S.-G. Kim, "Design considerations for MEMS scale piezoelectric mechanical vibration energy harvester," Integrated Ferroelectrics, vol. 71, no. 1, pp. 121-160, Jul. 2005.

[2] J. Twiefel and H. Westermann, "Survey on broadband techniques for vibration energy harvesting," Journal of Intelligent Material Systems and Structures, vol. 24, no. 11, pp. 1291-1302, Feb. 2013.

[3] S. Roundy and Y. Zhang, "Toward self-tuning adaptive vibration-based microgenerators," 2005, pp. 373-384.

[4] M. Lallart, S. R. Anton, and D. J. Inman, "Frequency Self-tuning Scheme for Broadband Vibration Energy Harvesting," Journal of Intelligent Material Systems and Structures, vol. 21, no. 9, pp. 897-906, Jun. 2010.

[5] R. G. Ballas, Piezoelectric multilayer beam bending actuators static and dynamic behavior and aspects of sensor integration. Berlin; New York: Springer, 2007.

[6] ]M. Géradin, Mechanical vibrations: theory and application to structural dynamics, 2nd ed. Chichester; New York: John Wiley, 1997.

[7] A. Erturk and D. J. Inman, "An experimentally validated bimorph cantilever model for piezoelectric energy harvesting from base excitations," Smart Materials and Structures, vol. 18, no. 2, p. 025009 , Feb. 2009., 